cloud in the constellation Draco which is roughly a degree in size and lies at least 300 parsecs away. It has been mapped in the characteristic radio light of neutral hydrogen as well as in infrared light, which is produced by interstellar dust. In the figure, the X-ray intensity is displayed as a false-colour image overlayed on a contour plot of the infrared map. As can be seen, the X-rays are dim in regions where the infrared is bright, indicating that the cloud is shadowing a significant portion of the X-ray back-ground. Burrows and Mendenhall find that roughly $60-70$ per cent of the $\mathrm{X}$-ray light must originate behind the cloud. Snowden et al. ${ }^{9}$ have also observed the Draco cloud using ROSAT in a scanning mode, and derive similar results.

Although these observations provide important new information, they may raise more questions than they answer. Burrows and Mendenhall argue that the apparent distant contribution to the background cannot be truly pervasive, as would be implied by a halo model, without coming into conflict with earlier observational constraints. For example, if the hot gas extended to similar distances in the direction of the galactic poles (where the amount of neutral gas is low), then the soft X-ray sky would be much brighter there than is observed. But at the very least, these data do prove that hot gas is present in the Galaxy at much larger distances than previously envisaged. Additional ROSAT shadowing experiments of other clouds will be required to determine whether the direction towards the Draco cloud is anomalous or typical of the X-ray sky.

Steven M. Kahn is in the Departments of Physics and Astronomy, and the Space Sciences Laboratory, University of California at Berkeley, California 94720, USA.

1. Burrows, D. N. \& Mendenhall, J. A. Nature 351, 629-631 (1991).

2. Bowyer, O. S., Field, G. B. \& Mack, J. E. Nature 217, 32-34 (1968).

3. McCammon, D. \& Sanders, W. T. A. Rev. Astr. Astrophys. 28, $657-688$ (1990)

4. Bunner, A. N. et al. Nature 223, 1222-1226 (1969).

5. Jakobsen, P. \&. Kahn, S. M. Astrophys. J. 309, 682-693 (1986).

6. Jahoda, K., McCammon, D. \& Lockman, F. J. Astrophys. J. 311, L57-L61 (1986).

7 Snowden, S. L Cox, D. P. McCammon, D. \& Sanders, W. T. Astrophys. J. 354, 211-219 (1990).

8. Martin, C. \& Bowyer, S. Astrophys. J. 350, 242-261 (1990).

9. Snowden, S. L., Mebold, U., Hirth, W., Herbstmeier U. \& Schmitt, J. H. M. M. Science 252, 1529-1532 (1991).

\title{
Brilliant - rock art and art rock in Australia
}

\section{Clive Gamble}

LEss than 25 years ago, prehistoric stone tools from Australia were described as "crude and rather colourless". Today, in other hands, they coruscate, casting light not only on their value in survival but also on changes in social values deduced from interpretations of their form and aesthetic appeal.

One such claim is now put forward by Taçon in the latest issue of Antiquity ${ }^{2}$. Taçon works in the Kakadu National Park of northern Australia, which is known for its spectacular rock art and which has also been the centre of excavations in the deposits beneath the paintings. Archaeologists have discovered cultural changes in the region over possibly 50,000 years. Moreover, long-term ethnographic enquiries among local Aboriginal communities have added a sacred dimension to the interpretation of stone tools, unearthed in the excavations, that is not possible in other parts of the world. The question for prehistorians is whether this potent Australian combination of rocks, rock art and religious knowledge can be more widely applied to shift the study of ancient stone tools away from just assessing their function to act as a means of investigating changes driven by symbolic behaviour.

Taçon characterizes the different properties of the common rock types, cherts and quartzites in the Kakadu excavations as, respectively, dull and shining. Almost any- where else this would sound like a story about pretty pebbles on the beach, but not in northern Australia where in an ethnographic study Morphy ${ }^{3}$ has identified these properties as the key to understanding the aesthetics of spiritual power. The sources of such power for the Yolngu people of eastern Arnhem Land (which lies to the east of Kakadu) come, ultimately, from the activities of the great ancestral beings of the spiritual Dreamtime. They are contained in objects which have the quality of bir'yun, or brilliance. Spear points made from glittering quartzite are a natural example, but brilliance can also be created. Nowhere is it better seen than in the quality captured in Yolngu bark paintings where the fine cross-hatched lines make the images shimmer.

Such power can also be released as tools are made by reducing nodules of stone. White and Jones ${ }^{4}$ learned this when studying the Ngilipitji quartzite quarry in eastern Arnhem land. The stones have power because of their brilliance, which is likened by the Yolngu to the shining fat of an animal. The special killing power of the stone points is accounted for by this perceived property of djukurr mirri, having fat.

Morphy argues that bir'yun is an effect that operates cross-culturally. It is an essential part of any aesthetic system simply because it produces effects that human beings find stimulating and appealing. The precise interpretation of its power through associations with objects will of course be culturally specific.

This line of argument may seem to license archaeologists to use alternative classifications of their stone tools - no more lists of scrapers and arrowheads but rather the proportions of rock types arranged on a scale from dull to glittering. Taçon attempts just such an analysis for the past 6,000 years of Kakadu prehistory. He points to the unsuitability of quartzite for making long flakes, and interprets its unexpected dominance as reflecting changes in symbolic behaviour in the region when shimmering rocks outnumber the dull but less intractable red cherts. Taçon also claims that there was an associated change to a more brilliant art style, underlying which were symbolic rather than functional reasons.

Such a magpie model of aesthetics still needs to be adequately tested. Claims for universals in cognitive behaviour to crack prehistoric art systems are a feature of the field ${ }^{5,6}$, but few of them enjoy unqualified support for long. I suspect the concept of brilliance will likewise fade, but not before such work has rightly highlighted the need to reassess the conventional approach to stone tools - in truth, the standard typologies and analyses have revealed little about past behaviour except our own claim to be the great classifiers.

The interest of the magpie model lies not in the glitter factor but in the attempt, either archaeologically or ethnographically, to link the tools with other aspects of the regional landscape and the culture it contains. This point is still most effectively argued by the 'functional' camp that Taçon criticizes. In an archaeological study of raw-material rationing in north Queensland, Hiscock ${ }^{7}$ shows how technology varies between regions with no change in the implement types. Variation lies in the processes by which flake artefacts are reduced from nodules. This in turn depends upon the suitability of raw material and its availability, so that as transport occurs shapes change in predictable ways - as others have noted, working with European materials ${ }^{8,9}$. By bringing a little colour to the argument, the result is an appreciation of the regional organization of mobile peoples as both sophisticated and subtle.

Clive Gamble is in the Department of Archaeology, University of Southampton, Southampton SO9 $5 \mathrm{NH}$, UK.

1. Clark, J. G. D. Economic Prehistory (Cambridge Univ. Press, 1989).

2. Taçon, P. Antiquity 65, 185-198 (1991).

3. Morphy, H. Man 24, 21-40 (1989).

4. Jones, R. \& White, N. in Archaeology with Ethnography (eds Meehan, B. \& Jones, R.) 51-87 (Australian National Univ., Canberra, 1988)

5. Leroi-Gourhan, A. Prehistorie de l'art Occidental (Mazenod Paris, 1965).

6. Lewis-Williams, J. D. \& Dowson, T. A. Curr. Anthropol. 29 201-245 (1988)

7. Hiscock, P. in Archaeology at Anzaas (ed. Ward, G.) 178190 (Canberra Arch. Soc., Canberra, 1984)

8. Kuhn, S. L. J. anthropol. Archaeol. 10, 76-106 (1991).

9. Rolland, N. \& Dibble, H. Am. Antiq. 55, 480-499 (1990). 